\title{
Anterior abdominal wall abscess caused by Streptococcus pneumoniae in a patient with self-inflicted stab injury: An unusual presentation
}

\author{
Shailesh Kumar, Sivaraman Umadevi, Joshy M Easow, Noyal Mariya Joseph, Sreenivasan \\ Srirangaraj, Kandha Kumari, Selvaraj Stephen
}

Department of Microbiology, Mahatma Gandhi Medical College and Research Institute, Pondicherry, India

\begin{abstract}
Streptococcus pneumoniae is a major cause of community-acquired pneumonia, otitis media, paranasal sinusitis, bacteremia and meningitis, as well as osteomyelitis and occasionally peritonitis. We report the case of a 25 -year-old female who had stabbed herself with a kitchen knife above the umbilicus 10 days prior to admission. Subsequently, she developed an anterior abdominal wall abscess caused by $S$. pneumoniae. This case is unusual as the focus was distant from the respiratory tract, the usual primary site of infection caused by this organism. Furthermore, the case assumes significance because it occurred in the absence of any typical risk factors for S. pneumoniae.
\end{abstract}

Key words: abdominal wall abscess; Streptococcus pneumoniae; stab injury

J Infect Dev Ctries 2011; 5(4):307-309.

(Received 12 July 2010 - Accepted 19 October 2010)

Copyright (C) 2011 Kumar et al. This is an open-access article distributed under the Creative Commons Attribution License, which permits unrestricted use, distribution, and reproduction in any medium, provided the original work is properly cited.

\section{Introduction}

Streptococcus pneumoniae is a normal inhabitant of the human upper respiratory tract [1]. The bacterium can cause pneumonia, usually of the lobar type, paranasal sinusitis, otitis media, or meningitis, the latter usually being secondary to one of the former infections [1]. It can also cause osteomyelitis, septic arthritis, endocarditis, peritonitis, cellulitis and brain abscesses $[1,2]$. S. pneumoniae is currently the primary cause of invasive bacterial disease in children and the elderly [1]. We report the case of a 25-year-old female who developed an anterior abdominal wall abscess caused by $S$. pneumoniae following a self-inflicted stab injury with a kitchen knife above the umbilicus. This case is unusual as the focus of the infection was distant from the respiratory tract, which is the usual primary site of infection caused by this organism. This case further assumes significance since it occurred in the absence of any of the typical risk factors for $S$. pneumoniae.

\section{Case report}

A 25-year-old female presented with a painful swelling in the periumbilical region which she had experienced for 10 days, and four to five episodes of bilious vomiting over three days. She gave a history of a suicide attempt 10 days previously, when she stabbed herself with a kitchen knife above the umbilicus, following which she was treated conservatively in another health-care centre and advised to undergo ultrasonography of the abdomen and computed tomography (CT), for which she came to our hospital (Mahatma Gandhi Medical College and Research Institute). On initial evaluation, she was afebrile with stable vitals. Abdominal examination revealed tenderness over the site of the injury and a subcutaneous anterior abdominal wall swelling, $5 \times 2$ $\mathrm{x} 3 \mathrm{~cm}$ which was firm in consistency. There was no discharge or organomegaly. Investigations revealed a significantly elevated white blood cell count of $252 \times$ $10^{3} / \mu 1$ with $85 \%$ neutrophils, $8 \%$ band forms and $7 \%$ lymphocytes, platelet count of $95 \times 10^{3} / \mu 1$, erythrocyte count of $2.7 \times 10^{6} / \mu \mathrm{l}$, hemoglobin level of $10.4 \mathrm{~g} / \mathrm{dl}$, hematocrit of $35 \%$, and a markedly elevated C-reactive protein level of $354 \mathrm{mg} / \mathrm{liter}$. Chest $\mathrm{X}$ ray of the patient was normal. Ultrasonography of the abdomen revealed cystic areas with unilateral echoes seen in the periumbilical region. The CT scan clearly showed an anterior abdominal wall abscess communicating with the intraabdominal region. Based on these findings, laparotomy and drainage of pus was planned. During the procedure, a pus-filled cavity (with about $20 \mathrm{ml}$ of pus) was found in the parietal wall. The pus was 
seen oozing out through a hole in the posterior rectus sheath and peritoneum into the peritoneal cavity. Intra-operatively, the greater curvature of the stomach was found to be oedematous and thickened; the stab wound was seen tracking down from the skin, tearing the posterior rectus sheath widely and entering the greater curvature of the stomach tangentially without producing any perforation. The supracolic area was filled with numerous flakes, while the infracolic area was free. Approximately 50 $\mathrm{ml}$ of pus was drained from the peritoneal cavity and a saline wash was performed. The anterior rectus and skin were closed after the procedure. The sample of abscess fluid revealed Gram-positive cocci in pairs with lanceolate appearance, morphologically resembling $S$. pneumoniae. The organism grew well on $5 \%$ sheep blood agar and chocolate plates at $37^{\circ} \mathrm{C}$ in a $\mathrm{CO}_{2}$ incubator. There were alpha-hemolytic, pitting colonies on the sheep blood agar and nonmucoid, pitting colonies with greenish discoloration on the chocolate agar. Colonies were catalase negative, susceptible to ethylhydrocuprein (optochin) (zone of inhibition $>14 \mathrm{~mm}$ ) and bile soluble. The biochemical profiles generated by the API 32 Strep system (bio-Merieux, New Delhi, India) identified the isolate as $S$. pneumoniae. The strain was serotyped by Neufeld's Quellung reaction, using type and factor sera and showed pneumococcal serotype 14. The isolate was sensitive to penicillin (MIC < $0.016 \mu \mathrm{g} / \mathrm{ml})$, vancomycin $(<0.38 \mu \mathrm{g} / \mathrm{ml})$, ciprofloxacin $(0.75 \mu \mathrm{g} / \mathrm{ml})$ and clindamycin $(<0.047$ $\mu \mathrm{g} / \mathrm{ml})$ according to the Clinical Laboratory Standards Institute guidelines [3]. The patient was treated with intravenous ciprofloxacin, ceftriaxone, and metronidazole for a total duration of ten days. Her condition improved dramatically and she became afebrile. She was advised to take ciprofloxacin and metronidazole orally for another 10 days and to return to the hospital for follow-up.

\section{Discussion}

$S$. pneumoniae may be either a colonizer of the skin and soft tissue or a pathogen. As a pathogen, it causes clinical diseases that vary widely in prognosis and severity. The isolation of $S$. pneumoniae from skin and soft tissue is an unusual finding with difficult clinical interpretation. Shahin and Lerner reported an immunocompetent patient with $S$. pneumoniae pneumonia and concomitant bacteriuria, who presented with abscesses in multiple soft tissue sites [4]. Green and Selinger reported a patient who presented with a urinary tract infection and soft tissue abscess caused by $S$. pneumoniae without any further focus of infection in the respiratory tract [5]. Most reported cases of $S$. pneumoniae soft tissue infection have involved cellulites that arose by direct inoculation from trauma, as in our case [6-8].

In a study performed by Garcia et al., of the 39 isolates of $S$. pneumoniae obtained from skin and soft tissue infections, the most frequent serotypes were 3 , 19, 11 and 23; however, in this case, serotype 14 was isolated [9]. Other predisposing factors for infections due to $S$. pneumoniae include immunosuppression and asplenia, which may also favor renal infections [1]. Michael and Cannon reported an asplenic patient with pneumococcal abscesses in multiple organs [10].

The case reported here assumes significance because it occurred in the absence of any of the typical risk factors for invasive pneumococcal disease, such as splenectomy, steroid use, diabetes mellitus, intravenous drug use, connective disorders or alcoholism [11]. In general, pneumococcal abscesses with or without secondary peritoneal involvement have a good prognosis, if prompt surgical drainage is performed and appropriate antibiotics are administered $[12,13]$. In our case the patient responded well to surgical drainage and intravenous antibiotic therapy, emphasizing the importance of correct microbiological diagnosis and treatment.

To conclude, although Streptococcus pneumoniae is frequently associated with human respiratory illnesses, only rarely it has been implicated in an abdominal wall abscess. Furthermore, our case assumes significance because it occurred in the absence of the characteristic risk factors.

\section{References}

1. Musher DM (1992) Infections caused by Streptococcus pneumoniae: clinical spectrum, pathogenesis, immunity, and treatment. Clin Infect Dis 14: 801-807.

2. Naktin J and DeSimone J (1999) Lumbar vertebral osteomyelitis with mycotic abdominal aortic aneurysm caused by highly penicillin-resistant Streptococcus pneumoniae. J Clin Microbiol 37: 4198-4200.

3. Clinical Laboratory Standards Institute (2007) Performance standards for antimicrobial susceptibility testing. Sixteenth informational supplement. CLSI document M100-S16. CLSI: Wayne, PA.

4. Shahin GS and Lerner SA (2002) Rare presentation of Streptococcus pneumoniae pneumonia with bacteremia and multiple subcutaneous abscesses. Eur J Clin Microbiol Infect Dis 21: 611-612. 
5. Green RF and Selinger DS (1980) Urinary tract infection and soft tissue abscess caused by Streptococcus pneumoniae. South Med J 73: 1550-1551.

6. Kragsbjerg P, Noren T and Soderquist B (1995) Deep softtissue infections caused by Streptococcus pneumoniae. Eur J Clin Microbiol Infect Dis 14: 1002-1004.

7. Lawlor MT, Crowe HM and Quintiliani R (1992) Cellulitis due to Streptococcus pneumoniae: case report and review. Clin Infect Dis 14: 247-250.

8. Patel M, Ahrens JC, Moyer DV and DiNubile MJ (1994) Pneumococcal soft-tissue infections: a problem deserving more recognition. Clin Infect Dis 19: 149-151.

9. Garcia-Lechuz JM, Ceuvas O and Castellares C (2007) Streptococcus pneumoniae skin and soft tissue infections: characterization of causative strains and clinical illness. Eur J Clin Microbiol Infect Dis 26: 247-253.

10. Michael MI and Cannon NJ (1974) Primary pneumococcal abscess in an asplenic host. Ala J Med Sci 11: 308-311.

11. Taylor SN and Saunders CV (1999) Unusual manifestations of invasive pneumococcal infection. Am J Med 107: 12S$24 \mathrm{~S}$.
12. Nakazato T, Kitahara M, Watanabe K, Kikuchi T, Imazu Y and Inoue K (1999) Pneumococcal psoas abscess. Intern Med 38: 63-66.

13. Capdevila O, Pallares R, Grau I, Tubau F, Linares J, Ariza J and Gudiol F (2001) Pneumococcal peritonitis in adult patients: report of 64 cases with special reference to emergence of antibiotic resistance. Arch Intern Med 161: $1742-1748$

\section{Corresponding author}

Dr. Shailesh Kumar

Associate Professor

Department of Microbiology

Mahatma Gandhi Medical College and Research Institute

Pillaiyarkuppam

Pondicherry - 607402

Telephone: +919944125839

Email: shaileshgar@yahoo.com

Conflict of interests: No conflict of interests is declared. 